\title{
In Vitro Evaluation of an Inverted End-to-End Equine Jejunojejunal Anastomosis Using Skin Staples
}

\author{
MARCO GANDINI, MedVet and ANDREA BERTUGLIA, MedVet, PhD
}

\begin{abstract}
Objective-To compare functional indices of end-to-end (EEA) jejunojejunal anastomosis using skin staples in horses with a 2-layer inverting hand-sewn technique.

Study Design-Experimental study.

Sample Population-Jejunal segments from 8 fresh equine cadavers.

Methods-For each bowel segment, 2 EEA anastomoses were created: one 2-layer hand-sewn and one 1-layer using skin staples. Time for anastomosis creation was recorded and compared. Lumen diameter of each anastomosis was measured on digital radiographs after intraluminal instillation of contrast medium and inflation of the jejunal segments to $14 \mathrm{~mm} \mathrm{Hg}$. Anastomotic indices (a compensated measure of stoma diameter) and bursting pressure were determined.

Results-EEA jejunal anastomosis using skin staples was significantly faster than use of a 2-layer hand-sewn technique. Anastomotic index, a measure of lumen size, was significantly larger with the skin-staple technique; however, the bursting pressure of stapled anastomoses was significantly less than for the hand-sewn technique, but the values were well above those reported for other anastomotic techniques.

Conclusions-An anastomotic technique using skin staples was easy to learn and perform, effective and faster, and mechanically comparable with a hand-sewn 2-layer technique.

Clinical Relevance-The staple technique could be beneficial in equine gastrointestinal surgery by reducing anastomosis time, although further in vivo studies are needed to establish clinical safety. (C) Copyright 2006 by The American College of Veterinary Surgeons
\end{abstract}

\section{INTRODUCTION}

A NASTOMOSES USING skin staples were initially used by military surgeons to create a single layer, inverting, end-to-end (EEA) anastomosis for treatment of intestinal gunshot injuries. ${ }^{1}$ Preliminary studies in pigs and clinical application in human patients have been reported. ${ }^{1-3}$ The method was easy to learn, safe, faster, and mechanically superior to conventional, inverting handsewn techniques. In dogs, a method for performing an everted, single layer, jejunojejunal EEA stapling technique resulted in a fast, safe, and mechanically comparable anastomosis with a routine hand-sewn pattern. ${ }^{4}$

Our purpose was to compare the functional indices of EEA jejunojejunal anastomoses created using either skin staples or a 2-layer hand-sewn inverting technique in equine jejunal specimens. We hypothesized that stapled anastomoses could be constructed more quickly, would have a larger lumen, and would be as strong as sutured anastomoses. To test this, we constructed EEA anastomoses in fresh jejunal specimens and measured anastomotic construction time, luminal diameter, and bursting pressure, and evaluated failure mode.

\section{MATERIALS AND METHODS}

\section{Jejunal Specimens}

Jejunal segments were collected from 8 slaughtered horses. Segments were harvested at least $2 \mathrm{~m}$ proximal to the ileocecal

From the Department of Animal Pathology, Faculty of Veterinary Medicine, University of Turin, Turin, Italy.

Presented as a poster at the 8th Equine Colic Research Symposium, Quebec City, August 2005.

Address reprint requests to Marco Gandini, MedVet, Dipartimento di Patologia Animale, Facoltà di Medicina Veterinaria di Torino, Via Leonardo da Vinci 44, 10095 Grugliasco (TO), Italy.

E-mail: marco.gandini $@$ unito.it.

Submitted January 2006; Accepted April 2006

(C) Copyright 2006 by The American College of Veterinary Surgeons

0161-3499/06

doi:10.1111/j.1532-950X.2006.00208.x 


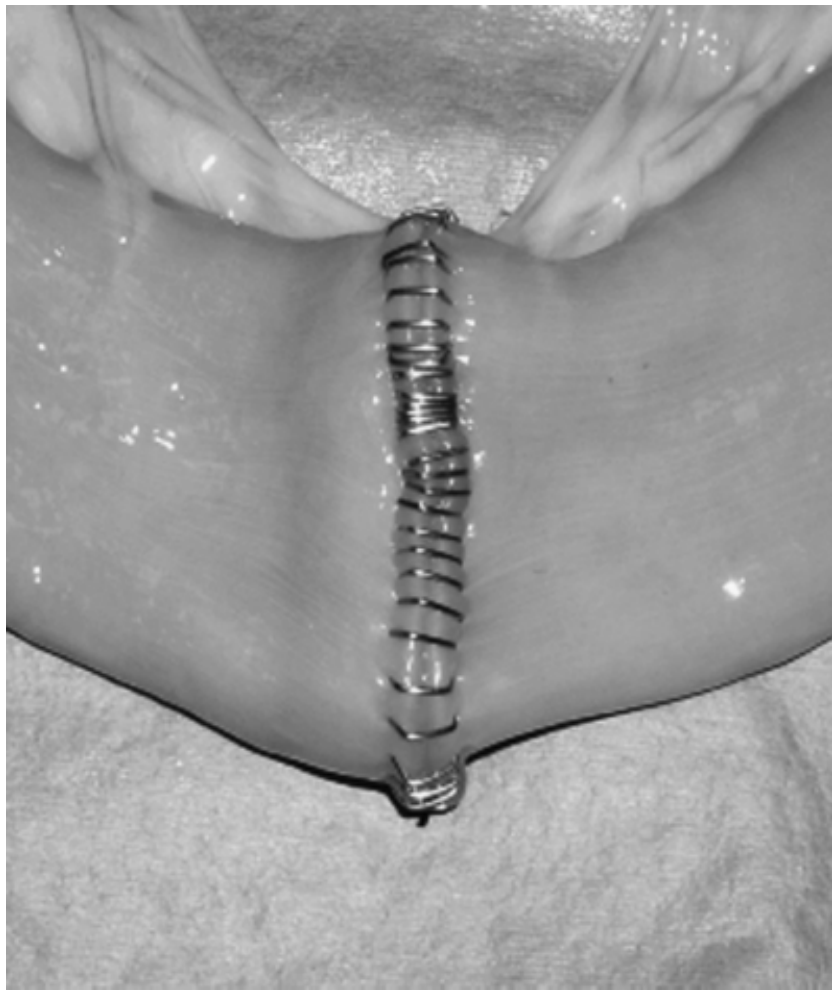

Fig 1. Anastomosis completed with skin staples. Staples may appear closer than $1 \mathrm{~mm}$ or farther apart because they were applied while the suture line was under tension, whereas the photograph was taken with the bowel relaxed.

valve and transported and stored at room temperature in $0.9 \%$ saline solution. Each segment was used within 4 hours of death to create 2 EEA anastomosis types: a 1-layer inverting anastomosis using skin staples (Weck Visistat ${ }^{\circledR}$ 35R, staple size $5.7 \times 3.9 \mathrm{~mm}$; Weck Teleflex Medica, Research Triangle Park, NC; Fig 1) and a hand-sewn double-layer inverting anastomoses. All anastomoses were performed by 1 surgeon (M.G.).

\section{Surgical Technique}

After cutting the jejunum with scissors at $\sim 60^{\circ}$ to the mesenteric border, the mesentery was stripped $5 \mathrm{~mm}$ back from the transected edges with a moistened sponge.

Stapled Anastomosis. Three single inverting horizontal mattress seromuscular sutures (2-0 polyglactin 910$)$ were inserted into each end as stay sutures; 1 suture was inserted on the mesenteric border and the other 2 sutures inserted so that there was approximately equidistant spacing between the 3 sutures around the circumference. Traction applied to the stay sutures produced slight inversion of the transected edges. Skin staples were then inserted $\sim 1-2 \mathrm{~mm}$ apart around the circumference, applying minimal pressure to the bowel surface, to produce an inverted pattern. Approximately 50-70 staples were used for each anastomosis.

Hand Sewn Anastomosis. A 2-layer inverting sutured anastomosis composed of a single continuous layer in the mucosa oversewn with a continuous Cushing pattern in the seromuscular layer using 3 metric polyglactin 910 was used as a control. After alignment of the 2 segments with 2-stay sutures inserted at the mesenteric and antimesenteric borders, the mucosal suture layer was interrupted half-way around the circumference to reduce the purse-string effect. Likewise, the Cushing pattern was also interrupted once around the circumference.

Time (minutes) to complete each anastomosis was recorded from insertion of the first stay suture to the insertion of the last suture or staple. After anastomosis completion, each bowel segment was transected $\sim 15 \mathrm{~cm}$ proximal and distal to the anastomosis and stored in $0.9 \%$ saline at room temperature before testing. Mechanical testing was conducted on all specimens within 1 hour of completion of the anastomosis.

\section{Lumen Diameter and Anastomotic Index}

Before biomechanical testing, each jejunal segment was rinsed with $0.9 \%$ saline solution. Then, the lumen was filled with a saline solution containing $10 \%$ of barium sulfate cream 113\% (Prontobario esofago, Bracco s.p.a, Milano, Italy), for 1 minute after which the fluid was milked out of the specimen. The segment ends were closed with plastic self-locking bands and the lumen was filled with air to an intraluminal pressure of $14 \mathrm{~mm} \mathrm{Hg}$. Specimens were radiographed with a digital system (Agfa Mimosa Vips 1206, Agfa-Gevaert N.V/S.A 96, Munich, Germany) and lumen diameter at the anastomotic site and at 2 sites each $5 \mathrm{~cm}$ distant from the anastomosis were measured on the radiographic images.

To account for interindividual horse variation, an anastomotic index was calculated with the formula: index $=2 \times$ anastomotic lumen diameter $(\mathrm{cm}) /($ distal lumen diameter $[\mathrm{cm}]+$ proximal lumen diameter $[\mathrm{cm}])$. This value is the ratio between the lumen diameter at the anastomotic site and the mean lumen diameter of proximal and distal sites.

\section{Biomechanical Testing}

Anastomotic leakage was evaluated by specimen inflation with air and subsequent immersion in a water tank. Bursting strength was determined for all specimens using a modified gas inflation tank test. A metal cannula connected to a compressed air tank was inserted into the lumen at 1 end of the specimen and a similar cannula inserted at the other end was connected to a calibrated mercury sphygmomanometer. The specimen was submerged in water, inflated with air at $1 \mathrm{~L} / \mathrm{min}$ until gas leaked from the bowel. Luminal pressures were continuously measured and recorded by digital camera. Review of recordings allowed determination of the peak pressure at specimen failure. Failure was confirmed by observation of gas bubbles leaking from the specimen and a decline in luminal pressure.

\section{Statistical Analysis}

Anastomosis construction time and anastomotic index for the 2 anastomosis types were compared using a paired 
Table 1. Summary Data for Construction Time, Bursting Pressure and Anastomotic Index for Hand sewn (HS) and Stapled (SS) Jejunal Anastomoses

\begin{tabular}{|c|c|c|c|c|c|c|}
\hline \multirow[b]{2}{*}{ Specimen } & \multicolumn{2}{|c|}{$\begin{array}{l}\text { Construction Time } \\
\text { (minutes) }\end{array}$} & \multicolumn{2}{|c|}{ Bursting Pressure $(\mathrm{mm} \mathrm{Hg})$} & \multicolumn{2}{|c|}{ Anastomotic Index } \\
\hline & HS & SS & HS & SS & HS & SS \\
\hline 2 & 15.93 & 6.70 & 170 & 95 & 0.69 & 0.75 \\
\hline 3 & 17.80 & 6.20 & 190 & 160 & 0.71 & 0.74 \\
\hline 4 & 17.25 & 7.53 & 185 & 145 & 0.8 & 0.82 \\
\hline 7 & 17.08 & 6.83 & 165 & 160 & 0.76 & 0.81 \\
\hline 8 & 18.25 & 6.50 & 180 & 120 & 0.72 & 0.8 \\
\hline Mean & 17.24 & 6.63 & 180.38 & 133.13 & 0.72 & 0.77 \\
\hline SD & 0.86 & 0.47 & 11.20 & 46.44 & 0.04 & 0.04 \\
\hline
\end{tabular}

Student's t-test. Bursting pressure values were compared between techniques using a Wilcoxon's matched pair test. All statistical analyses were performed with commercially available software (Graphpad InStat ${ }^{\circledR}$ version 3.05 for Windows 95/NT, GraphPad Software, San Diego, CA) with significance set at $P \leq .05$. Results are reported as mean \pm SEM.

\section{RESULTS}

Hand-sewn anastomoses had more pronounced inversion whereas the stapled anastomosis had a more bulky appearance. The mean $( \pm$ SEM $)$ completion time for stapled anastomosis $(6.63 \pm 0.17$ minutes $)$ was significantly faster than for hand-sewn anastomosis $(17.24 \pm 0.31$ minutes; $P<.0001$; Table 1). The mean anastomotic index for stapled anastomoses $(0.77 \pm 0.01)$ was significantly higher than for the hand-sewn anastomosis $(0.72 \pm 0.01$; $P<.0001$ ). Only 1 stapled anastomosis leaked air after completion; none of the hand-sewn anastomoses leaked after completion.

All but 1 hand-sewn anastomosis failed at the mesenteric attachment, at sites distant from the anastomosis, at a mean pressure of $180.83 \pm 3 \mathrm{~mm} \mathrm{Hg}$. The specimen that failed at the anastomotic site leaked where the 2 continuous sutures merged in proximity to the antimesenteric site. The mean bursting pressure for stapled anastomoses $(133.3 \pm 19.09 \mathrm{~mm} \mathrm{Hg})$ was significantly lower than for the sutured anastomosis $(P=.0078)$. Two stapled anastomoses failed at mesenteric sites distant from the anastomosis (Fig 2), whereas the other specimens failed at different locations within the anastomosis.

After distension, serosal tears were associated with each staple in all stapled anastomoses but were not associated with specimen failure.

\section{DISCUSSION}

We found that an EEA jejunojejunal anastomosis created by use of skin staples could be performed more rapidly than a 2-layer hand-sewn anastomosis and had less reduction in luminal diameter but lower bursting pressure. The recorded bursting pressures for both anastomosis types were markedly higher than intraluminal pressures recorded in horses with bowel distention. Hand-sewn anastomoses typically failed on the mesenteric border in the intermesenteric space away from the anastomosis, whereas stapled anastomoses typically failed between the anastomosed edges.

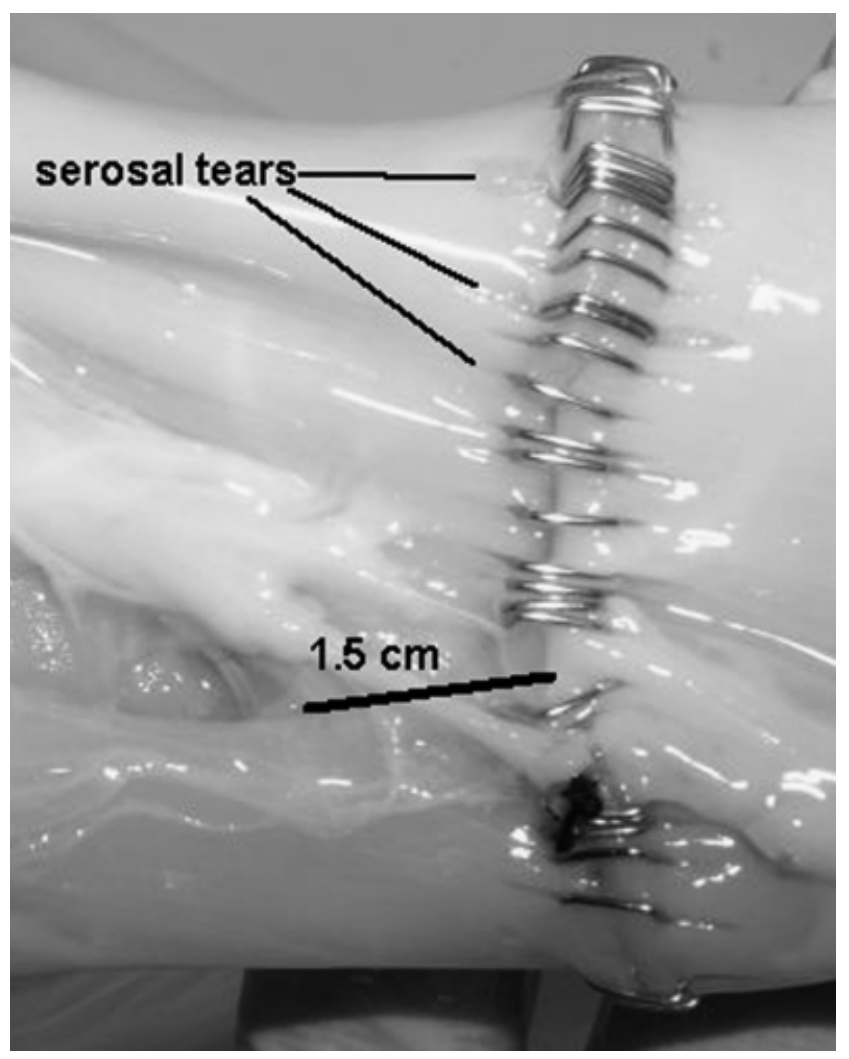

Fig 2. Failure in the intermesenteric region at a distant site from anastomosis in 1 stapled specimen. 
Jejunojejunal EEA anastomoses are commonly performed in equine intestinal surgery. One approach is a 2layer inverting hand-sewn pattern, a technique that is demanding in terms of surgical skills and time. Use of conventional intestinal staplers in horses has been limited by cost and the dimensions of available instruments. The EEA stapling devices commonly used in humans and small animals have been used in ponies ${ }^{5}$ but cannot be used in adult horses because the dimensions are too small compared with horse luminal diameter. ${ }^{5,6}$ Linear thoraco-abdominal (TA) staplers have been used to construct everting or inverting triangulated EEA anastomoses; however, both techniques have some disadvantages. The everted anastomosis leaves contaminated mucosa exposed to the abdominal cavity, whereas the inverting pattern is demanding in terms of skills and time. ${ }^{7,8}$ Linear gastrointestinal (GIA, gastrointesinal anastomosis, or ILA, intestinal linear anastomosis) staple devices have been used successfully to create true side to side or functional EEA jejunojejunal anastomoses in horses, ${ }^{9,10}$ although their cost can be a limiting factor and their use is also not without complications. ${ }^{1-13}$ Nevertheless, a major advantage of intestinal stapling instruments is the precision by which staples are applied that ensure an even distribution thus reducing the chance of anastomotic leakage. With the use of skin staplers, even distribution, is a function of the surgeon's skill, although placing the tip of the instrument against the previously placed staple can facilitate achieving a standardized distance between staples.

The skin-staple technique that we describe was easy to learn and perform. The single anastomoses that leaked air just after completion was the first performed. Subsequent attention to stay suture positioning resulted in optimal edge inversion and improved placement of the stay sutures and staples resulted in an airtight seal at completion. In an in vivo study in dogs, ${ }^{4}$ all stapled anastomoses leaked air after completion but did not leak ingesta or result in postoperative complications. Some of our stapled anastomoses were stronger than intact intestinal wall, and all accommodated intraluminal pressures higher than those reported for other stapled anastomosis. ${ }^{14}$ Failure of most anastomoses (stapled and hand sewn) occurred on the mesenteric border in the intermesenteric space at a site distant from the anastomosis, highlighting the importance of the serosa as a force-resisting layer. After distension, serosal tears occurred in stapled anastomoses as reported for other 1-layer techniques ${ }^{15}$ but were not associated with specimen failure.

Exposed foreign material used to create anastomoses is of concern for potential adhesion formation. The likelihood of adhesions to skin staples in the equine abdomen is unknown but could potentially be prevented with the use of anti-adhesion membranes ${ }^{16,17}$ or surgical techniques. ${ }^{18}$ Complications directly related to skin staple use in the gastrointestinal tract have not been reported in dogs, ${ }^{4,19}$ pigs, ${ }^{2,20,21}$ or humans. ${ }^{3}$ In vivo studies are needed to evaluate the abdominal response in horses. Staple migration can occur after remodeling of the anastomosis. In dogs, ${ }^{19} 8$ months after skin-stapled closure of enterotomies, one-third of the staples were no longer visible on abdominal radiographs. The staples had apparently migrated into the bowel lumen and been evacuated in feces. In a skin-stapled EEA anastomosis study in $\operatorname{dogs},{ }^{4}$ staples were completely covered by fibrous connective tissue at 21 days.

Disposable skin staplers are readily available and are produced by several different manufacturers. Our preliminary testing indicated that regular size $(5.7 \mathrm{~mm} \times 3.9 \mathrm{~mm})$ staples were adequate for horses because they resulted in a better approximation of the edges in an inverting EEA jejunojejunal anastomosis. Other staple sizes are now available and may be better suited to this application. Hemorrhage from enterotomy sites may be of concern ${ }^{23}$ as skin staples do not provide hemostasis because of their design. Thus, if used for anastomosis, it would be important to ensure adequate hemostasis of the transected bowel ends before stapling. Skin staples are cost-effective compared with intestinal stapling devices and the technique we describe reduces the amount of mucosa exposed compared with an everted EEA-stapled anastomosis technique.

Seemingly, the main advantages of our method are speed of construction and reduced bowel handling compared with traditional hand-sewn techniques, factors that may be beneficial in reducing postoperative morbidity. Care with adequate hemostasis of transected ends before anastomosis and precise positioning facilitated by stay suture placement will be important to prevent leakage. Based on our results, skin staple devices may be useful to create technically simple and rapid EEA anastomoses in equine jejunum, and clinical evaluation of the technique seems warranted.

\section{REFERENCES}

1. Howell GP, Morgans BT, Ryan JM, et al: Assessment of the use of disposable skin staplers in bowel anastomoses to reduce laparotomy time in penetrating ballistic injury to the abdomen. Ann R Coll Surg Engl 73:87-90, 1991

2. Edwards DP, Galbraith KA.: Colonic anastomosis in the presence of fecal peritonitis using a disposable skin stapler. J Invest Surg 11:267-274, 1998

3. Fraser I: Intestinal anastomosis with a skin stapler: a safe and efficient method in humans. Br J Surg 81:665-667, 1994

4. Coolman BR, Ehrhart N, Pijanowski G, et al: Comparison of skin staples with sutures for anastomosis of the small intestine in dogs. Vet Surg 29:293-302, 2000 
5. Robertson-Smith RG, Adams SB: Evaluation of an end-toend stapling instrument for anastomosis of the small intestine in the pony. Vet Surg 16:99, 1987

6. Freeman DE: Surgery of the small intestine. Vet Clin North Am Equine Pract 13:261-301, 1997

7. Bristol DG, Cullen J: Use of a linear stapling device to construct an inverted, triangulated, end-to-end anastomosis of the equine jejunum. Cornell Vet 79:88-93, 1989

8. Sullins KE, Stashak T, Mero K: Evaluation of intestinal staples for end-to-end anastomosis of the small intestine of the horse. Vet Surg 14:87-91, 1985

9. Baxter GM, Hunt RJ, Tyler D, et al: Sutured end-to-end and stapled side-to-side jejunal anastomosis in the horse. Vet Surg 21:47-55, 1992

10. Coatney RW, Adams SB: Evaluation of a linear stapling instrument for side-to-side anastomosis of small intestine in the pony (abstract). Vet Surg 18:63, 1989

11. Latimer FG, Blackford JT, Valk N, et al: Closed one-stage functional end-to-end jejunojejunostomy in horses with use of linear stapling equipment. Vet Surg 27:17-28, 1998

12. Mackey VS, Pascoe JR, Peterson PR: A potential technique error in stapled side-to-side anastomosis of the small intestine of the horse. Vet Surg 16:189-192, 1987

13. Frankeny RL, Wilson DA, Messer NT, et al: Jejunal intussusception: a complication of functional end-to-end stapled anastomosis in two ponies. Vet Surg 24:515-517, 1995

14. Bickers RJ, Blackford JT, Eiler H, et al: A comparison of the mechanical strength of two stapled anastomosis techniques for equine small intestine. Vet Surg 31:104-110, 2002

15. Nieto JE, Snyder JR: Comparison of a single versus a double layer hand sewn end-to-end anastomosis in the jejunum of horses. Proceedings of the 8th International Equine Colic Research Symposium, Quebec City, QC, Canada, 2005, pp 192-193

16. Fogle CA, Elce YE, Little D, et al. Association of sodium carboxymethylcellulose and a bioresorbable hyaluronatecarboxymethylcellulose membrane with postoperative factors in horses with small intestinal surgery: 33 cases (2003-2004). Proceedings of the 8th International Equine Colic Research Symposium, Quebec City, QC, Canada, 2005, pp 179-180

17. Gandini M, Bertuglia A: Mesenteric flap to prevent adhesions after small intestinal end to end anastomosis in the horse. Proceedings of the 8th International Equine Colic Research Symposium 2005, Quebec City, QC, Canada, pp 94-96

18. Dawson DL, Coil JA, Jadali M, et al: Use of skin staplers in experimental gastrointestinal injuries. J Trauma 32:204 209, 1992

19. Fackler ML, Breteu JPL, Sendowski ICP, et al: Perforating wounds of the abdomen by the modern assault rifle. J Trauma (China) 6(Suppl): 192-199, 1990

20. Coolman B, Ehrart N, Marretta SM: Use of skin staples for rapid closure of gastrointestinal incisions in the treatment of canine linear foreign bodies. J Am Anim Hosp Assoc 36:542-547, 2000

21. Doyle AJ, Freeman DE, Rapp H, et al: Life-threatening hemorrhage from enterotomies and anastomosis in 7 horses. Vet Surg 32:553-558, 2003

22. Eggleston RB, Mueller E, Quandt JE, et al: Use of a hyaluronate membrane for jejunal anastomosis in horses. Am J Vet Res 62:1314-1319, 2001 